\title{
DYNAMICAL BEHAVIOUR OF A DRINKING EPIDEMIC MODEL
}

\author{
SWARNALI SHARMA AND G. P. SAMANTA*
}

\begin{abstract}
In this paper we have constructed a mathematical model of alcohol abuse which consists of four compartments corresponding to four population classes, namely, moderate and occasional drinkers, heavy drinkers, drinkers in treatment and temporarily recovered class. Basic reproduction number $R_{0}$ has been determined and sensitivity analysis of $R_{0}$ indicates that $\beta_{1}$ (the transmission coefficient from moderate and occasional drinker to heavy drinker) is the most useful parameter for preventing drinking habit. Stability analysis of the model is made using the basic reproduction number. The model is locally asymptotically stable at disease free or problem free equilibrium (DFE) $E_{0}$ when $R_{0}<1$. It is found that, when $R_{0}=1$, a backward bifurcation can occur and when $R_{0}>1$, the endemic equilibrium $E^{*}$ becomes stable. Further analysis gives the global asymptotic stability of DFE under some conditions. Our important analytical findings are illustrated through computer simulation. Epidemiological implications of our analytical findings are addressed critically.
\end{abstract}

AMS Mathematics Subject Classification : 93B35.

Key words and phrases : Basic reproduction number, Sensitivity, Local and global Stability, Forward and Backward bifurcations.

\section{Introduction}

Alcohol abuse now becomes an epidemic. It is characterized by the sufferer having a pattern of drinking excessively despite the negative effects of alcohol on their work, health, educational and social life. The National Institute on Alcohol Abuse and Alcoholism [21, 22] estimates that 18 million Americans suffer from alcohol abuse, specially most beginning by their mid teens. Serious drinking often often starts in adolescence; approximately $40 \%$ of alcoholics develop their first symptoms between 15 and 19 years of age [13, 35]. Alcohol related problems cost so much that it affects the economic structure of the countries $[8,9,10,11$,

Received October 23, 2012. Revised December 24, 2012. Accepted January 4, 2013. * Corresponding author.

(C) 2013 Korean SIGCAM and KSCAM. 
$13,19,25]$. Over-consumption of alcohol is now the third leading cause of death all over the world. One study links alcohol to 1 in every 25 deaths worldwide a nd the $5 \%$ of years lived with disability are attributable to alcohol consumption [23]. Long-term use of alcohol of damaging nearly every organ and system in the body. The World Health Organization (WHO) estimates that alcohol is supposed to cause about 60 types of diseases and injury like 20-30\% of esophageal cancer, liver cancer, cirrhosis of the liver, homicide, epileptic seizures and motor vehicle accidents worldwide [23]. There are two major forms of intervention policy of alcohol abuse: (i) prevention initiation into alcohol abuse and (ii) rehabilitation of alcohol abusers. Among the many problem confronting these programmes the most important is the very high rates of relapse after treatment. The National Institute on Alcohol Abuse and Alcoholism [21, 22] estimates that up to $70 \%$ of treated alcohol abusers relapse after treatment which is indeed a big problem. Therefore prevention and control efforts that include treatment and education programmes are in need of improvement so that the rate of relapse from treatment can be reduced. Mathematical studies can be effective as guides to the evaluation, testing and implementation of strategies over short or long time scales for chronic relapsing diseases such as alcohol addiction, drug abuse etc. There are many mathematical models for epidemic diseases like HIV, SARS, gonorrhea, dengue $[3,4,14,15,16,26,27,29,31]$ etc. But the mathematical models on alcohol and drug abuse are very small in number though they have been referred to in terms of epidemics.

Two very interesting models have recently been proposed. One for treating heroin users proposed by White and Comiskey [36]. The model involves three classes of population, namely susceptible, heroin users and heroin users in treatment. And a similar one for those with alcohol problems proposed by Sánchez et. al. [30]. This model also divides the mathematical problem into three classes, namely susceptible, alcoholics and alcoholics undergoing treatment. In fact, the two types of model are very similar. The one of Sánchez et. al. [30] defers from that of White and Comiskey [36] only in that, she assumes the same death/ removal rate for each of the three classes, whereas White and Comiskey [36] correctly allow the drug users and those in treatment to have enhanced death rates. After that the heroin epidemic model of White and Comiskey [36] was revisited and developed by many researchers like Mulone and Straughan [20], Liu and Zhang [18], Nyabadza and Hove-Musekwa [24], Samanta [28] etc. There are also many useful mathematical epidemic models which have generated useful insights about the role of behaviour on the transmission dynamics of sexually transmitted diseases like gonorrhea [6, 16], HIV [31] or communicable diseases such as SARS [12] etc.

In this paper, we have developed an alcohol abuse model. We have divided the mathematical problem into four classes, namely, moderate and occasional drinkers, heavy drinkers, drinkers in treatment and temporarily recovered class. Next we have found the basic reproduction number $R_{0}[32,33]$. Then the stability analysis of the model is discussed using the basic reproduction number. It 
is observed that the model is locally asymptotically stable at disease free equilibrium $E_{0}$ when $R_{0}<1$. When $R_{0}=1$, a backward bifurcation can occur, i.e., although $R_{0}$ may be less than 1 , an endemic equilibrium exists. That means the backward bifurcation occurs because of the insufficient capacity for treatment policies. As a result the drinkers in treatment come to the direct contact of heavy drinkers and they re-enter into the heavy drinkers class. When $R_{0}>1$ endemic equilibrium exists and becomes stable and the DFE becomes unstable. Next we have found the conditions of global asymptotic stability of $E_{0}$ and the conditions of local asymptotic stability of $E^{*}$ by Routh-Hurwitz criterion [17]. Next we have illustrated some of the key findings through numerical simulations followed by conclusions. Our aim of introducing this four compartmental alcohol abuse model is to identify the parameters of interest for further study. It also will be very useful for informing and assisting policy-makers in targeting prevention and treatment resources for maximum effectiveness.

\section{Mathematical Model}

In this section we have constructed a mathematical model of alcohol abuse. The adult human population is divided into four different classes, namely, moderate and occasional drinkers with density $S(t)$, heavy drinkers with density $D(t)$, drinkers in treatment with density $T(t)$, temporarily recovered class with density $R(t)$ at time t.

The following figure represents the flow of individuals from one class to the other:

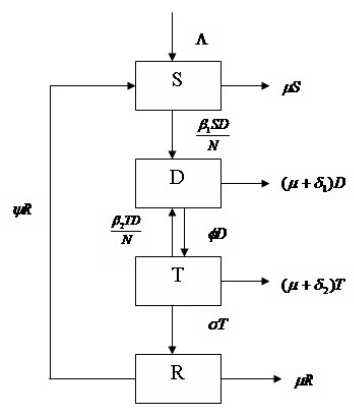

Fig.1. Flow diagram of the alcohol abuse model

The model can be presented by the following set of ordinary differential equations:

$$
\begin{aligned}
\frac{d S}{d t} & =\Lambda-\beta_{1} S(t) \frac{D(t)}{N}-\mu S(t)+\psi R(t) \\
\frac{d D}{d t} & =\beta_{1} S(t) \frac{D(t)}{N}+\beta_{2} T(t) \frac{D(t)}{N}-\left(\mu+\delta_{1}+\phi\right) D(t) \\
\frac{d T}{d t} & =\phi D(t)-\beta_{2} T(t) \frac{D(t)}{N}-\left(\mu+\delta_{2}+\sigma\right) T(t) \\
\frac{d R}{d t} & =\sigma T(t)-(\mu+\psi) R(t)
\end{aligned}
$$


with initial densities

$$
S(0)>0, D(0) \geq 0, T(0) \geq 0, R(0) \geq 0 .
$$

The model parameters are described below:

$\Lambda$ : Recruitment rate of moderate and occasional drinkers,

$\beta_{1}$ : The transmission coefficient from moderate and occasional drinkers to heavy drinkers,

$\beta_{2}$ : The transmission coefficient from drinker in treatment to heavy drinker,

$\mu$ : Natural death rate of population,

$\delta_{1}$ : Drinking related death rate of heavy drinkers,

$\delta_{2}$ : Drinking related death rate of drinkers in treatment,

$\phi:$ The proportion of drinkers who enter treatment,

$\sigma$ : Recovery rate of drinkers in treatment,

$\psi$ : The proportion of recovered class who enter to moderate and occasional drinkers class again.

Clearly the model involves the following assumptions:

(i) The population we are dealing is isolated and closed. This results in the total population size remaining constant. Every member of the population corresponds to one of the subgroups and these subgroups are mutually exclusive. This means that $N=S(t)+D(t)+T(t)+R(t)$, where $\mathrm{N}$ is the total size of the population,i.e., $\Lambda=\mu S+\left(\mu+\delta_{1}\right) D+\left(\mu+\delta_{2}\right) T+\mu R$, i.e., the population is assumed to be of constant size within the modelling time.

(ii) All members of the population mix homogeneously. This means that each individual has an equal chance of becoming a heavy drinker.

(iii) The heavy drinking is passed to moderate and occasional drinkers by adequate contact with Heavy drinkers not in treatment.

(iv) Heavy drinkers not in treatment are infectious to moderate and occasional drinkers and to drinkers in treatment.

(v) Drinkers in treatment are not infectious to moderate and occasional drinkers.

(vi) The drinkers in treatment most commonly relapse due to contact with heavy drinkers who are not in treatment.

(vii) Those who stop drinking alcohol enter to the temporarily recovered class and one part of which relapse to the moderate and occasional drinkers' class.

\section{Basic Properties}

\subsection{Invariant Region.}

Theorem 1. If we assume that $N$ is dependent on time $t$, the feasible region $G$ defined by

$$
G=\left\{(S(t), D(t), T(t), R(t)) \in \mathbb{R}_{+}^{4}: 0<N \leq \frac{\Lambda}{\mu}\right\}
$$

with initial conditions $S(0)>0, D(0) \geq 0, T(0) \geq 0, R(0) \geq 0$, is positively invariant. 
Proof. Adding the equations of the system (1) we obtain

$$
\frac{d N}{d t}=\Lambda-\mu N-\delta_{1} D(t)-\delta_{2} T(t) \leq \Lambda-\mu N
$$

The solution $N(t)$ of the differential equation (3) has the following property,

$$
0 \leq N(t) \leq N(0) e^{-\mu t}+\frac{\Lambda}{\mu}\left(1-e^{-\mu t}\right)
$$

where $N(0)$ represents the sum of the initial values of the variables. As $t \rightarrow$ $\infty, 0 \leq N \leq \frac{\Lambda}{\mu}$. So if $N(0) \leq \frac{\Lambda}{\mu}$ then $\lim _{t \rightarrow \infty} N(t) \leq \frac{\Lambda}{\mu}$. This means that $\frac{\Lambda}{\mu}$ is the upper bound of $N$. On the other hand, if $N(0)>\frac{\Lambda}{\mu}$, then $N(t)$ will decrease to $\frac{\Lambda}{\mu}$. This means that if $N(0)>\frac{\Lambda}{\mu}$, then the solution $(S(t), D(t), T(t), R(t))$ enters $G$ or approach it asymptotically. Hence it is positively invariant under the flow induced by the system (1). Thus in $G$, the model (1) is well-posed epidemiologically and mathematically. Hence it is sufficient to study the dynamics of the model in $G$.

\subsection{Non-negativity of Solutions.}

Theorem 2. Every solution of system (1) with initial conditions (2) exists in the interval $[0, \infty)$ and $S(t)>0, D(t) \geq 0, T(t) \geq 0, R(t) \geq 0$, for all $t \geq 0$.

Proof. Since the right hand side of system (1) is completely continuous and locally Lipschitzian on $C$, the solution $(S(t), D(t), T(t), R(t))$ of (1) with initial conditions $(2)$ exists and is unique on $[0, \xi)$, where $0<\xi \leq+\infty$. It follows from the 1st equation of the system (1) that,

$$
\frac{d S}{d t} \geq \Lambda-\left[\beta_{1} \frac{D(t)}{N}+\mu\right] S(t)
$$

We thus have,

$$
\frac{d}{d t}\left[S(t) \exp \left\{\mu t+\frac{1}{N} \int_{0}^{t} \beta_{1} D(s) d s\right\}\right] \geq \Lambda \exp \left\{\mu t+\frac{1}{N} \int_{0}^{t} \beta_{1} D(s) d s\right\} .
$$

Hence

$S(t) \exp \left\{\mu t+\frac{1}{N} \int_{0}^{t} \beta_{1} D(s) d s\right\}-S(0) \geq \int_{0}^{t} \Lambda \exp \left\{\mu t+\frac{1}{N} \int_{0}^{t} \beta_{1} D(s) d s\right\} d t$,

so that

$$
\begin{aligned}
& S(t) \geq S(0) \exp \left[-\left\{\mu t+\frac{1}{N} \int_{0}^{t} \beta_{1} D(s) d s\right\}\right]+\exp \left[-\left\{\mu t+\frac{1}{N} \int_{0}^{t} \beta_{1} D(s) d s\right\}\right] \\
& {\left[\int_{0}^{t} \Lambda \exp \left\{\mu t+\frac{1}{N} \int_{0}^{t} \beta_{1} D(s) d s\right\} d t\right]>0 . }
\end{aligned}
$$

From the 2nd equation of (1), we have

$$
\frac{d D}{d t} \geq-\left(\mu+\delta_{1}+\phi\right) D(t) \Rightarrow D(t) \geq D(0) \exp \left[-\left(\mu+\delta_{1}+\phi\right) t\right] \geq 0
$$


Similarly, from the 3rd equation of (1), we have

$$
\begin{aligned}
\frac{d T}{d t} & \geq-\left[\beta_{2} \frac{D(t)}{N}+\left(\mu+\delta_{2}+\sigma\right)\right] T(t) \\
\Rightarrow & T(t) \geq T(0) \exp \left[-\left\{\left(\mu+\delta_{2}+\sigma\right) t+\frac{1}{N} \int_{0}^{t} \beta_{2} D(s) d s\right\}\right] \geq 0 .
\end{aligned}
$$

Similarly, from the 4 th equation of (1), we have

$$
\frac{d R}{d t} \geq-(\mu+\psi) R(t) \Rightarrow R(t) \geq R(0) \exp [-(\mu+\psi) t] \geq 0 .
$$

Therefore, we can see that $S(t)>0, D(t) \geq 0, T(t) \geq 0, R(t) \geq 0, \quad \forall t \geq 0$. This completes the proof.

\section{The Basic Reproduction Number $R_{0}$}

Basic reproduction number $R_{0}[32,33]$ is defined as the number of heavy drinkers produced when a single heavy drinker is introduced into moderate and occasional drinkers' population. In this proposed model, the basic reproduction number is the transmission coefficient from moderate and occasional drinker to heavy drinker divided by the sum of the natural death rate of the population, the drinking related death rate of heavy drinkers who are not in treatment and the proportion of individuals who enter treatment, i.e.,

$$
R_{0}=\frac{\beta_{1}}{\mu+\delta_{1}+\phi} .
$$

\section{Sensitivity Analysis of $R_{0}$}

To examine the sensitivity of $R_{0}$ to each of its parameters, following normalized forward sensitivity index with respect to each of the parameters is computed $[1]$.

$$
\begin{gathered}
A_{\beta_{1}}=\frac{\frac{\partial R_{0}}{R_{0}}}{\frac{\partial \beta_{1}}{\beta_{1}}}=\frac{\beta_{1}}{R_{0}} \frac{\partial R_{0}}{\partial \beta_{1}}=\beta_{1}\left(\frac{\mu+\delta_{1}+\phi}{\beta_{1}}\right)\left(\frac{1}{\mu+\delta_{1}+\phi}\right)=1 \\
A_{\mu}=\frac{\frac{\partial R_{0}}{R_{0}}}{\frac{\partial \mu}{\mu}}=\frac{\mu}{R_{0}} \frac{\partial R_{0}}{\partial \mu}=\left|\frac{-\mu}{\mu+\delta_{1}+\phi}\right|<1, \\
A_{\delta_{1}}=\frac{\frac{\partial R_{0}}{R_{0}}}{\frac{\partial \delta_{1}}{\delta_{1}}}=\frac{\delta_{1}}{R_{0}} \frac{\partial R_{0}}{\partial \delta_{1}}=\left|\frac{-\delta_{1}}{\mu+\delta_{1}+\phi}\right|<1 . \\
A_{\phi}=\frac{\frac{\partial R_{0}}{R_{0}}}{\frac{\partial \phi}{\phi}}=\frac{\phi}{R_{0}} \frac{\partial R_{0}}{\partial \phi}=\left|\frac{-\phi}{\mu+\delta_{1}+\phi}\right|<1,
\end{gathered}
$$

From the above discussion we observe that the basic reproduction number $\left(R_{0}\right)$ is most sensitive to changes in $\beta_{1}$. If $\beta_{1}$ will increase $R_{0}$ will also increase with same proportion and if $\beta_{1}$ will decrease $R_{0}$ will also decrease in same proportion. $\mu, \delta_{1}$ and $\phi$ have an inversely proportional relationship with $R_{0}$, 
so an increase in any of them will cause a decrease in $R_{0}$. The increase in either of the rates $\mu$, the natural death rate of the population and $\delta_{1}$, the drinking related death rate of the heavy drinkers not in treatment, is neither ethical nor practical. So our aim is to focus on one of two parameters: either $\phi$, the proportion of drinkers who enter treatment or $\beta_{1}$, the transmission rate from moderate and occasional drinker to heavy drinker. As $R_{0}$ is more sensitive to changes in $\beta_{1}$ than $\phi$, it seems sensible to focus efforts on the reduction of $\beta_{1}$. In other words, this sensitivity analysis tells us that prevention is better than treatment, which means efforts to increase prevention are more effective in controlling the spread of habitual drinkers than efforts to increase the numbers of individuals accessing treatment.

\section{Stability of Drinking Free or Problem Free Equilibrium, $E_{0}$ when

$$
R_{0}<1
$$

We now use $R_{0}$ to determine the existence of equilibria for the system.In this section we will study the local stability behavior of the model system (1) at the drug free equilibrium (DFE) $E_{0}\left(\frac{\Lambda}{\mu}, 0,0,0\right)$.

Now, the variational matrix of system (1) at $E_{0}\left(\frac{\Lambda}{\mu}, 0,0,0\right)$ is given by

$$
V\left(E_{0}\right)=\left[\begin{array}{cccc}
-\mu & -\beta_{1} & 0 & \psi \\
0 & \beta_{1}-\left(\mu+\delta_{1}+\phi\right) & 0 & 0 \\
0 & \phi & -\left(\mu+\delta_{2}+\sigma\right) & 0 \\
0 & 0 & \sigma & -(\mu+\psi)
\end{array}\right]
$$

Therefore, eigen values of the characteristic equation of $V\left(E_{0}\right)$ are $\lambda_{1}=-\mu, \lambda_{2}=$ $\beta_{1}-\left(\mu+\delta_{1}+\phi\right), \lambda_{3}=-\left(\mu+\sigma+\delta_{2}\right), \lambda_{4}=-(\mu+\psi)$. Here, $\lambda_{1}, \lambda_{3}$ and $\lambda_{4}$ are clearly real and negative. Also as $R_{0}<1$, then

$$
\beta_{1}<\mu+\delta_{1}+\phi
$$

and therefore $\lambda_{2}$ is also real and negative. Therefore the system (1) shows local asymptotic stability at $E_{0}\left(\frac{\Lambda}{\mu}, 0,0,0\right)$. So, we arrive to the following result:

Theorem 3. The problem free equilibrium $E_{0}$ of the model system (1) is locally asymptotically stable if $R_{0}<1$.

\section{Analysis at $R_{0}=1$}

In this section, we discuss the stability of heavy drinking persistent equilibrium or problem persistent equilibrium and investigate the possibility of occurring backward bifurcation $[2,14,31,32,33,34,37]$ due to existence of multiple equilibrium. To analyze it for the system (1), we use the center manifold theory [5]. To implement this method, we first change the variables of the model equations (1) so that $S=x_{1}, D=x_{2}, T=x_{3}, R=x_{4}$ with $\frac{d x_{1}}{d t}=f_{1}, \frac{d x_{2}}{d t}=f_{2}, \frac{d x_{3}}{d t}=$ $f_{3}, \frac{d x_{4}}{d t}=f_{4}$. 
Thus system (1) becomes:

$$
\begin{aligned}
& f_{1}=\Lambda-\frac{\beta_{1} x_{1} x_{2}}{N}-\mu x_{1}+\psi x_{4} \\
& f_{2}=\frac{\beta_{1} x_{1} x_{2}}{N}+\frac{\beta_{2} x_{3} x_{2}}{N}-\left(\mu+\delta_{1}+\phi\right) x_{2} \\
& f_{3}=\phi x_{2}-\frac{\beta_{2} x_{3} x_{2}}{N}-\left(\mu+\delta_{2}+\sigma\right) x_{3} \\
& f_{4}=\sigma x_{3}-(\mu+\psi) x_{4}
\end{aligned}
$$

We choose $\beta_{1}^{*}=\beta_{1}$ as the bifurcation parameter,particularly as it has been shown in equation (5) that $R_{0}$ is more sensitive to change in $\beta_{1}$ than in its other parameters. If we consider $R_{0}=1$, then we obtain,

$$
\beta_{1}^{*}=\mu+\delta_{1}+\phi .
$$

Now, the Jacobian of the linearized system (6) using identity (7) at problem free equilibrium $E_{0}$ when $\beta_{1}^{*}=\beta_{1}$ is given by,

$$
J\left(\beta_{1}^{*}\right)=\left[\begin{array}{cccc}
-\mu & -\beta_{1}^{*} & 0 & \psi \\
0 & 0 & 0 & 0 \\
0 & \phi & -\left(\mu+\delta_{2}+\sigma\right) & 0 \\
0 & 0 & \sigma & -(\mu+\psi)
\end{array}\right]
$$

The matrix (8) has eigenvalues $\left(0,-\mu,-\left(\mu+\delta_{2}+\sigma\right),-(\mu+\psi)\right)^{T}$, which meets the requirement of simple zero eigenvalue and the others having negative real part. We can thus use the center manifold theory [5] to analyze the dynamics of system (6). The right eigenvector associated with zero eigenvalue is given by, $\omega=\left(\omega_{1}, \omega_{2}, \omega_{3}, \omega_{4}\right)^{T}$, where

$$
\begin{array}{llrl}
\omega_{1} & =\frac{1}{\mu}\left\{\psi-\frac{\beta_{1}^{*}\left(\mu+\delta_{2}+\sigma\right)(\mu+\psi)}{\phi \sigma}\right\} \omega_{4}, & \omega_{2}=\frac{\left(\mu+\delta_{2}+\sigma\right)(\mu+\psi)}{\phi \sigma} \omega_{4} \\
\omega_{3}=\frac{(\mu+\psi)}{\sigma} \omega_{4}, & \omega_{4}=1
\end{array}
$$

with $\omega_{4}$ free. Further, $J\left(\beta_{1}^{*}\right)$ has a corresponding left eigen vector $\nu=\left(\nu_{1}, \nu_{2}, \nu_{3}, \nu_{4}\right)$, where

$$
\nu_{1}=0, \quad \nu_{2}=1, \quad \nu_{3}=0 \text { and } \nu_{4}=0
$$

with $\nu_{2}$ free. In order to establish the local stability of $E^{*}$, we use the following theorem.

Theorem 4 ([7]). Consider the following general system of ordinary differential equations with a parameter $\varphi$,

$$
\frac{d x}{d t}=f(x, \varphi), \quad f: R^{n} \times R \rightarrow R \text { and } f \in C^{2}\left(R^{n} \times R\right),
$$

where 0 is an equilibrium of the system that is $f(0, \varphi)=0, \forall \varphi$ and assume: 
A1. $A=D_{x} f(0,0)=\left(\frac{\partial f_{i}}{\partial x_{j}}(0,0)\right)$ is linearization matrix of the system (9) around the equilibrium 0 with $\varphi$ evaluated at 0 . Zero is a simple eigenvalue of $A$ and all other eigenvalues of $A$ have negative real parts;

A2. Matrix $A$ has a right eigenvector $u$ and a left eigenvector $\nu$ corresponding to the zero eigenvalue.

Let $f_{k}$ be the $k$-th component of $f$ and

$$
a=\sum_{k, i, j=1}^{n} \nu_{k} u_{i} u_{j} \frac{\partial^{2} f_{k}}{\partial x_{i} \partial x_{j}}(0,0), \quad b=\sum_{k, j=1}^{n} \nu_{k} u_{i} \frac{\partial^{2} f_{k}}{\partial x_{i} \partial \varphi}(0,0) .
$$

The local dynamics of (9) around 0 are totally governed by a and $b$.

(i) $a, b>0$. When $\varphi<0$ with $|\varphi| \ll 1,0$ is locally asymptotically stable, and there exists a positive unstable equilibrium; when $0<\varphi \ll 1,0$ is unstable and there exists a negative and locally asymptotically stable equilibrium,

(ii) $a<0, b<0$. When $\varphi<0$ with $|\varphi| \ll 1$, 0 is unstable; when $0<\varphi \ll 1,0$ is locally asymptotically stable, and there exists a positive unstable equilibrium,

(iii) $a>0, b<0$. When $\varphi<0$ with $|\varphi| \ll 1,0$ is unstable, and there exists a locally asymptotically stable negative equilibrium; when $0<\varphi \ll 1$, 0 is stable, and a positive unstable equilibrium appears,

(iv) $a<0, b>0$. When $\varphi$ changes from negative to positive, 0 changes its stability from stable to unstable. Correspondingly a negative unstable equilibrium becomes positive and locally asymptotically stable.

The computation of $a$ and $b$ are necessary to apply the Theorem 1 .

In particular, since $\nu_{1}=\nu_{3}=\nu_{4}=0$,

$$
a=\nu_{2} \sum_{i, j=1}^{4} \omega_{i} \omega_{j} \frac{\partial^{2} f_{2}}{\partial x_{i} \partial x_{j}}(0,0) \text { and } b=\nu_{2} \sum_{i=1}^{4} \omega_{i} \frac{\partial^{2} f_{2}}{\partial x_{i} \partial \beta_{1}}(0,0)
$$

For the system (6), the associated non-zero partial derivatives at the problem free equilibrium are given by:

$\frac{\partial^{2} f_{2}}{\partial x_{1} \partial x_{2}}=\frac{\partial^{2} f_{2}}{\partial x_{2} \partial x_{1}}=\frac{\mu\left(\mu+\delta_{1}+\phi\right)}{\Lambda}, \frac{\partial^{2} f_{2}}{\partial x_{2} \partial x_{3}}=\frac{\partial^{2} f_{2}}{\partial x_{3} \partial x_{2}}=\frac{\beta_{2} \mu}{\Lambda}, \frac{\partial^{2} f_{2}}{\partial x_{2} \partial \beta_{1}}=1$.

It thus follows that,

$$
a=\frac{2 \mu \omega_{2}}{\Lambda}(X-\Gamma),
$$

where

$$
\begin{gathered}
X=\frac{\psi}{\mu}\left(\mu+\delta_{1}+\phi\right)+\frac{\beta_{2}}{\sigma}(\mu+\psi), \\
\Gamma=\frac{\left(\mu+\delta_{1}+\phi\right)^{2}\left(\mu+\delta_{2}+\sigma\right)(\mu+\psi)}{\phi \sigma \mu}
\end{gathered}
$$

and $b=\omega_{2}>0$. 
Hence the sign of $a$ depends on the values of $X$ and $\Gamma$, so that if $X>\Gamma$, then $a>0$ and if $X<\Gamma$, then $a<0$ while $b>0$ always. Thus, we have the following result:

Theorem 5. If $X>\Gamma$, then the system (1) has a backward bifurcation at $R_{0}=1$, otherwise if $X<\Gamma$, then it undergoes forward bifurcation and the endemic equilibrium is locally asymptotically stable for $R_{0}>1$, but close to one.

\section{Existence of Endemic Equilibrium $E^{*}\left(S^{*}, D^{*}, T^{*}, R^{*}\right)$ when $R_{0}>1$}

In this section, we analyze the existence of non-trivial endemic equilibrium of system (1). At an endemic equilibrium, disease is present and the followings hold:

$$
S>0, D>0, T>0, R>0, \frac{d S}{d t}=\frac{d D}{d t}=\frac{d T}{d t}=\frac{d R}{d t}=0 .
$$

Solving the equations of system (1) at equilibrium state we get,

$$
S^{*}=\frac{N\left\{b_{1} b_{2}+\left(\mu+\delta_{1}\right) \beta_{2} D^{*}\right\}}{\beta_{1}\left(b_{2}+\beta_{2} D^{*}\right)}, T^{*}=\frac{N \phi D^{*}}{\left(b_{2}+\beta_{2} D^{*}\right)}, R^{*}=\frac{N \sigma \phi D^{*}}{(\mu+\psi)\left(b_{2}+\beta_{2} D^{*}\right)}
$$

where

$$
b_{1}=\mu+\delta_{1}+\phi, \quad b_{2}=N\left(\mu+\delta_{2}+\sigma\right) .
$$

Now, putting the values of $S^{*}, T^{*}, R^{*}$ into the first equation of (1) and simplifying we obtain,

$$
a_{1}\left(D^{*}\right)^{2}+a_{2} D^{*}+a_{3}=0
$$

where

$$
\begin{aligned}
& a_{1}=\beta_{1} \beta_{2}(\mu+\psi)\left(\mu+\delta_{1}\right), \\
& a_{2}=(\mu+\psi)\left\{\beta_{1} b_{1} b_{2}+N \mu \beta_{2}\left(\mu+\delta_{1}\right)-\Lambda \beta_{1} \beta_{2}\right\}-\beta_{1} N \psi \sigma \phi, \\
& a_{3}=(\mu+\psi)\left(N \mu b_{1} b_{2}-\Lambda \beta_{1} b_{2}\right) .
\end{aligned}
$$

Obviously $a_{1}$ is always positive. However, the signs of $a_{2}$ and $a_{3}$ are not obvious although it is known that $\beta_{1}>\mu+\delta_{1}+\phi$ as $R_{0}>1$. Now using Descartes' rule of signs in equation (12) we obtain:

(i) if $a_{2}>0, a_{3}>0$, then there is no change of sign, so there exists no positive root of equation (12),

(ii) if $a_{2}>0, a_{3}<0$, there exists only one positive root of equation (12),

(iii) if $a_{2}<0, a_{3}<0$, there exists only one positive root of equation (12),

(iv) if $a_{2}<0, a_{3}>0$, there exists two or no positive root of equation (12).

Therefore, if $a_{3}$ is negative, then there exists at least one positive value of $D^{*}$, i.e., at least one non-trivial endemic equilibrium.

Summarizing the previous discussions we come to the following result:

Theorem 6. If $a_{3}$ in equation (12) is negative, then there exists at least one non-trivial endemic equilibrium of the system (1). 
Observation: If we take $\beta_{2}=0$, then

$$
a_{1}=0, a_{2}=(\mu+\psi) \beta_{1} b_{1} b_{2}-\beta_{1} N \psi \sigma \phi, a_{3}=(\mu+\psi)\left(N \mu b_{1} b_{2}-\Lambda \beta_{1} b_{2}\right) .
$$

Then the equation (12) becomes,

$$
a_{2} D^{*}+a_{3}=0 .
$$

Therefore, there exists only one root of the above equation, which is given by

$$
D^{*}=-\frac{a_{3}}{a_{2}} \text {. }
$$

Now, if $a_{2}<0$ or $a_{3}<0$ then the above equation has an unique positive root $D^{*}$. This implies that there is a unique endemic equilibrium point of the system (1) when $\beta_{2}=0$ and $a_{2}<0$ or $a_{3}<0$. Therefore in this case there is no existence of backward bifurcation as there is a unique endemic equilibrium of the system (1) when $\beta_{2}=0$ and $a_{2}<0$ or $a_{3}<0$.

Therefore, we can conclude that the backward bifurcation occurs because of the insufficient capacity for treatment policies. As a result the drinkers in treatment come to the direct contact of heavy drinkers and they re-enter into the heavy drinkers class.

\section{Global Stability Analysis of Disease Free or Problem Free Equilibrium $E_{0}$}

Let us define,

$$
L=P\left(S-S^{*}-S^{*} \ln \frac{S}{S^{*}}\right)+Q D+M T+U R,
$$

where $P, Q, M, U$ are positive constants to be chosen later. Here $L(S, D, T, R) \geq$ 0 and $L\left(S^{*}, D^{*}, T^{*}, R^{*}\right)=0$. Differentiating $L$ along the solutions of system (1) with respect to $t$, we get

$$
\begin{aligned}
\frac{d L}{d t}= & P \frac{S-S^{*}}{S} \frac{d S}{d t}+Q \frac{d D}{d t}+M \frac{d T}{d t}+U \frac{d R}{d t} \\
= & P \frac{S-S^{*}}{S}\left[\Lambda-\beta_{1} \frac{S D}{N}-\mu S+\psi R\right]+Q\left[\beta_{1} \frac{S D}{N}+\beta_{2} \frac{T D}{N}-\left(\mu+\delta_{1}+\phi\right) D\right] \\
& +M\left[\phi D-\beta_{2} \frac{T D}{N}-\left(\mu+\delta_{2}+\sigma\right) T\right]+U[\sigma T-(\mu+\psi) R] \\
= & P \frac{S-S^{*}}{S}\left[\mu S+\left(\mu+\delta_{1}\right) D+\left(\mu+\delta_{2}\right) T+\mu R\right]-P \beta_{1} \frac{D}{N}\left(S-S^{*}\right)-P \mu\left(S-S^{*}\right) \\
& +\frac{P R \psi}{S}\left(S-S^{*}\right)+Q \beta_{1} \frac{S D}{N}+Q \beta_{2} \frac{T D}{N}-Q D\left(\mu+\delta_{1}+\phi\right)+M \phi D \\
& -M \beta_{2} \frac{T D}{N}-M T\left(\mu+\delta_{2}+\sigma\right)+U T \sigma-U R(\mu+\psi) \\
= & -D \mu(Q-P)-D \delta_{1}(Q-P)-T \mu(M-P)-T \delta_{1}(M-P)-R \mu(U-P) \\
& -\beta_{1} \frac{S D}{N}(P-Q)-R \psi(U-P)-\beta_{2} \frac{T D}{N}(M-Q)-D \phi(Q-M)-T \sigma(Q-M) \\
& +\frac{P D \beta_{1} S^{*}}{N}-\frac{P R \psi S^{*}}{S}-\frac{P S^{*}}{S}\left[\left(\mu+\delta_{1}\right) D+\left(\mu+\delta_{2}\right) T+\mu R\right]
\end{aligned}
$$


Now, we choose $P=Q=M=U$ then simplifying we get,

$$
\frac{d L}{d t}=-P S^{*} D\left[\frac{\mu+\delta_{1}}{S}-\frac{\beta_{1}}{N}\right]-\frac{P S^{*}}{S}\left(\mu+\delta_{2}\right) T-\frac{P R S^{*}}{S}(\mu+\psi)
$$

Now, $\frac{d L}{d t}<0$ if $\left(\mu+\delta_{1}\right) \geq \beta_{1}$, then $\frac{d L}{d t}$ is negative definite and consequently, $L$ is a Lyapunov function $[18,26]$ with respect to all solutions in the interior of the positive orthant.

Summarizing the above discussions we come to the following result:

Theorem 7. If $R_{0}<1$ and $\left(\mu+\delta_{1}\right) \geq \beta_{1}$ then $E_{0}$ is globally asymptotically stable.

\section{Stability Analysis of Endemic Equilibrium $E^{*}\left(S^{*}, D^{*}, T^{*}, R^{*}\right)$ using Routh-Hurwitz Criterion}

The variational matrix of system (1) at $E^{*}$ is given by,

$$
V\left(E^{*}\right)=\left[\begin{array}{cccc}
m_{11} & m_{12} & 0 & m_{14} \\
m_{21} & 0 & m_{23} & 0 \\
0 & m_{32} & m_{33} & 0 \\
0 & 0 & m_{43} & m_{44}
\end{array}\right]
$$

where,

$$
\begin{aligned}
& m_{11}=-\frac{\beta_{1} D^{*}}{N}-\mu, \quad m_{12}=-\frac{\beta_{1} S^{*}}{N}, \quad m_{14}=\psi, \quad m_{21}=\frac{\beta_{1} D^{*}}{N}, \quad m_{23}=\frac{\beta_{2} D^{*}}{N}, \\
& m_{32}=\phi-\frac{\beta_{2} T^{*}}{N}, \quad m_{33}=-\frac{\beta_{2} D^{*}}{N}-\left(\mu+\delta_{2}+\sigma\right), \quad m_{43}=\sigma, \quad m_{44}=-(\mu+\psi) .
\end{aligned}
$$

Therefore, the characteristic equation of $V\left(E^{*}\right)$ is,

$$
\lambda^{4}+A_{1} \lambda^{3}+A_{2} \lambda^{2}+A_{3} \lambda+A_{4}=0,
$$

where,

$$
\begin{aligned}
& A_{1}=-m_{11}-m_{33}-m_{44}, \\
& A_{2}=m_{11} m_{33}+m_{11} m_{44}+m_{33} m_{44}-m_{12} m_{21}-m_{23} m_{32}, \\
& A_{3}=m_{12} m_{21} m_{33}+m_{12} m_{21} m_{44}+m_{11} m_{23} m_{32}+m_{23} m_{32} m_{44}-m_{11} m_{33} m_{44}, \\
& A_{4}=-m_{12} m_{21} m_{33} m_{44}-m_{11} m_{23} m_{32} m_{44}-m_{21} m_{14} m_{32} m_{43} .
\end{aligned}
$$

By the Routh-Hurwitz criterion [17], it follows that, all eigenvalues of the characteristic equation (15) has negative real part if and only if

$$
A_{1}>0, \quad A_{4}>0, \quad B=A_{1} A_{2}-A_{3}>0, \quad B A_{3}-A_{1}^{2} A_{4}>0 .
$$

Theorem 8. $E^{*}$ is locally asymptotically stable if and only if the above inequalities of (16) are satisfied. 


\section{Numerical Simulations}

In this section we present computer simulation of some important results of our discussions of the system (1). Beside verification of our analytical findings, these numerical solutions are very important from practical point of view.

We first consider the case when $R_{0}=0.482759<1$ using the parameter values given in Table 1. For different initial conditions the dynamics of the model is presented in fig.2. The figure shows that only moderate and occasional drinkers' population exists $(S=1.6)$ and the populations of heavy drinkers, drinkers in treatment and temporarily recovered population declines to zero $(D=0, T=0, R=0)$, i.e., approaches the disease free or problem free equilibrium (DFE). It shows that DFE is locally asymptotically stable whenever $R_{0}<1$. This numerical verification supports the result stated in Theorem 3 (art.6) on the stability of DFE.

Further using the parameter values given in Table 2, we consider the case when $R_{0}=1.25>1$. For different initial conditions the dynamics of the model is presented in fig.3. The figure shows that moderate and occasional drinkers' population, heavy drinkers, drinkers in treatment and temporarily recovered population all exist $\left[\left(S^{*}, D^{*}, T^{*}, R^{*}\right)=(11.4291,0.2513,0.7846,2.2417)\right]$, i.e., the population of drinkers tends to drinking persistent equilibrium (DPE) or endemic equilibrium and the DFE $\left[E_{0}=(36,0,0,0)\right]$ exists but becomes unstable when $R_{0}>1$. This indicates that irrespective of the initial conditions the population of heavy drinkers eventually settles at endemic equilibrium with increasing time and the problem free equilibrium became unstable when $R_{0}>1$, which supports our analytical results. In this case $X=0.329$ and $\Gamma=1.36102$, i.e., $X<\Gamma$, so $a<0$ (see 11,11a,11b, art.7). Therefore, it also shows the forward bifurcation of system (1) which is good agreement with Theorem 5.

Further using the parameter values given in Table 3, we consider the case when $R_{0}=0.75<1$. For different initial conditions the dynamics of the model is presented in fig.4. The figure shows that in this case, there exists three equilibria of the system (1), among them problem free equilibrium $E_{0}(36,0,0,0)$ and an endemic equilibrium $E^{*}(17.4074,5.8495,1.9973,0.1598)$ are stable and the other endemic equilibrium $E_{1}(25.1106,2.8411,1.7854,0.1428)$ is unstable. Here $X=13.015$ and $\Gamma=8.32$, i.e., $X>\Gamma$, so $a>0$ (see 11,11a,11b, art.7). Therefore, it also shows the backward bifurcation of system (1) which is also in good agreement with Theorem 5 .

TABLE 1

\begin{tabular}{|c|c||c|c|}
\hline Parameter & Values & Parameter & Values \\
\hline$\Lambda$ & 0.4 & $\phi$ & 0.7 \\
\hline$\beta_{1}$ & 0.7 & $\psi$ & 0.1 \\
\hline$\beta_{2}$ & 0.3 & $\delta_{1}$ & 0.5 \\
\hline$\mu$ & 0.25 & $\delta_{2}$ & 0.3 \\
\hline$\sigma$ & 0.09 & & \\
\hline
\end{tabular}




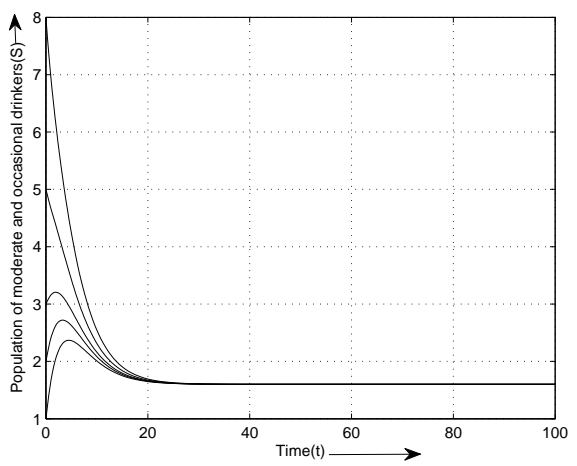

Fig.2a. Time series plot of the moderate and occasional drinkers for $R_{0}=$ $0.482759<1$ with various initial conditions, parameter values are given in Table 1.

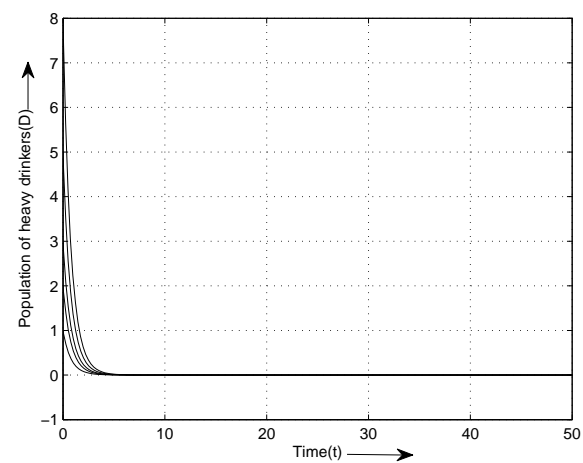

Fig.2b. Time series plot of heavy drinkers for $R_{0}=0.482759<1$ with various initial conditions, parameter values are given in Table 1.

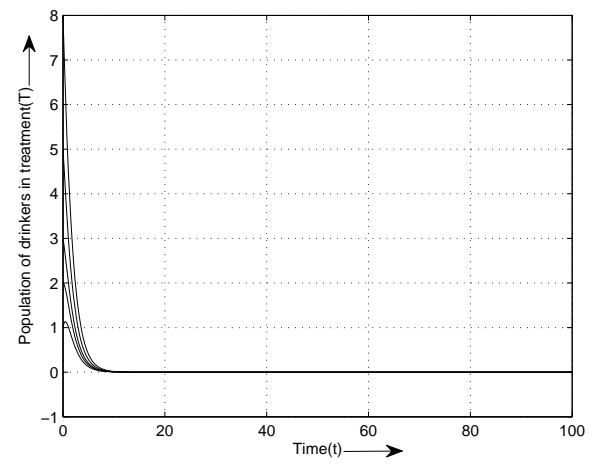

Fig.2c. Time series plot of drinkers in treatment for $R_{0}=0.482759<1$ with various initial conditions, parameter values are given in Table 1. 


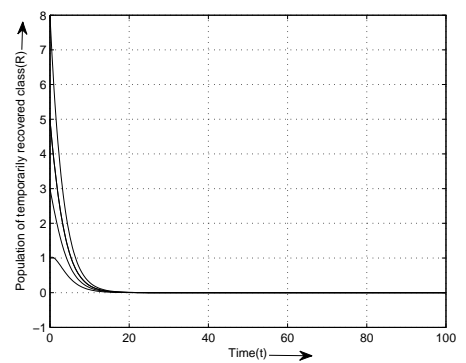

Fig.2d. Time series plot of temporarily recovered class for $R_{0}=0.482759<1$ with various initial conditions, parameter values are given in Table 1.

TABLE 2

\begin{tabular}{|c|c||c|c|}
\hline Parameter & Values & Parameter & Values \\
\hline$\Lambda$ & 0.4 & $\phi$ & 0.5 \\
\hline$\beta_{1}$ & 0.7 & $\psi$ & 0.01 \\
\hline$\beta_{2}$ & 0.3 & $\delta_{1}$ & 0.035 \\
\hline$\mu$ & 0.025 & $\delta_{2}$ & 0.03 \\
\hline$\sigma$ & 0.1 & & \\
\hline
\end{tabular}

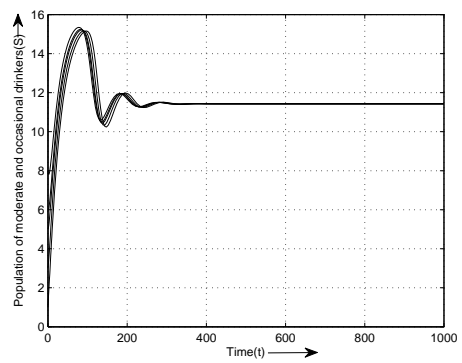

Fig.3a. Time series plot of moderate and occasional drinkers for $R_{0}=1.25>1$ with various initial conditions, parameter values are given in Table 2.

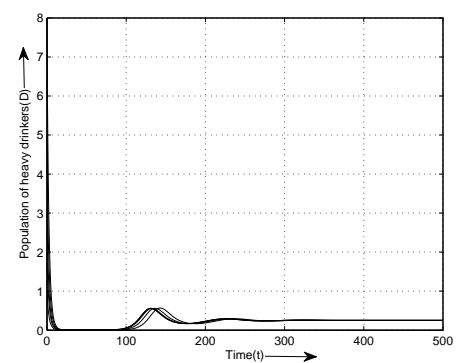

Fig.3b. Time series plot of heavy drinkers for $R_{0}=1.25>1$ with various initial conditions, parameter values are given in Table 2. 


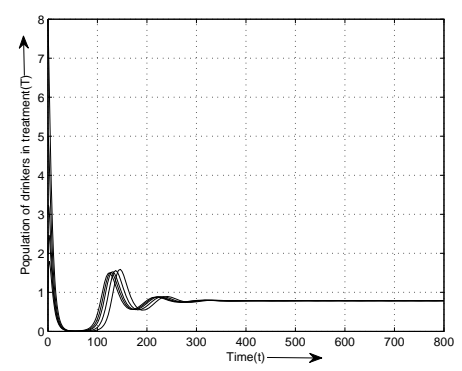

Fig.3c. Time series plot of the drinkers in treatment for $R_{0}=1.25>1$ with various initial conditions, parameter values are given in Table 2.

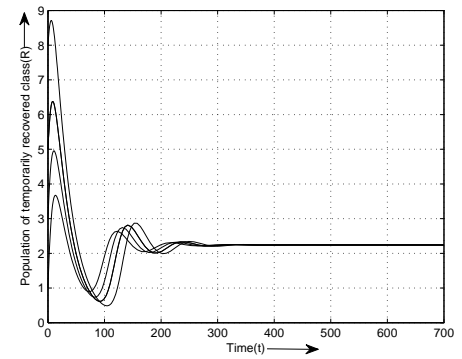

Fig.3d. Time series plot of temporarily recovered class for $R_{0}=1.25>1$ with various initial conditions, parameter values are given in Table 2.

TABLE 3

\begin{tabular}{|c|c||c|c|}
\hline Parameter & Values & Parameter & Values \\
\hline$\Lambda$ & 0.9 & $\phi$ & 0.1 \\
\hline$\beta_{1}$ & 0.12 & $\psi$ & 0.1 \\
\hline$\beta_{2}$ & 0.99 & $\delta_{1}$ & 0.035 \\
\hline$\mu$ & 0.025 & $\delta_{2}$ & 0.03 \\
\hline$\sigma$ & 0.01 & & \\
\hline
\end{tabular}

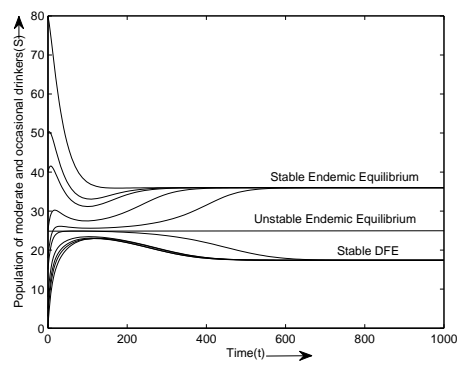

Fig.4a. Time series plot of moderate and occasional drinkers for $R_{0}=0.75<1$ with various initial conditions, parameter values are given in Table 3. 


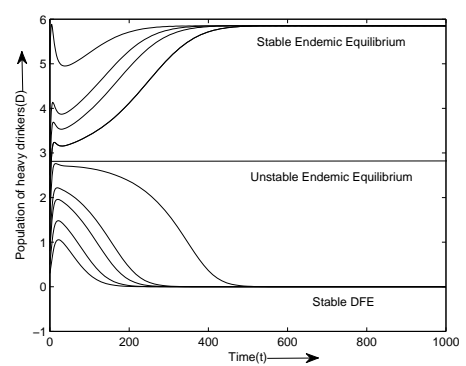

Fig.4b. Time series plot of heavy drinkers for $R_{0}=0.75<1$ with various initial conditions, parameter values are given in Table 3.

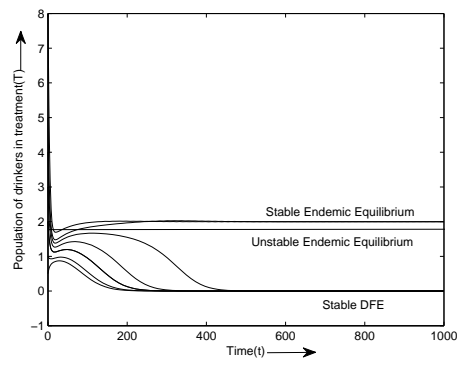

Fig.4c. Time series plot of the drinkers in treatment for $R_{0}=0.75<1$ with various initial conditions, parameter values are given in Table 3.

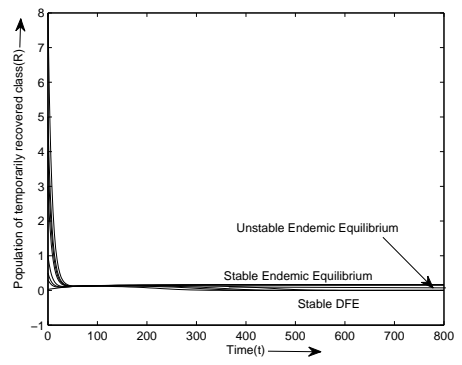

Fig.4d. Time series plot of temporarily recovered class for $R_{0}=0.75<1$ with various initial conditions, parameter values are given in Table 3.

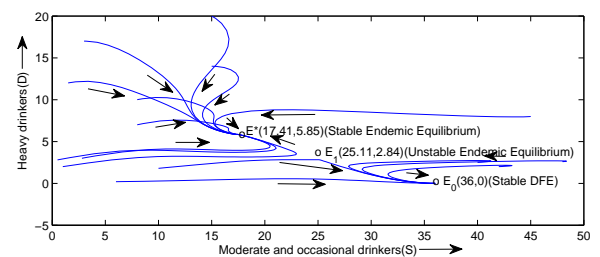

Fig.4e. Moderate and occasional drinkers and heavy drinkers' $S$-D plane projection of the solution for $R_{0}=0.75<1$ with various initial conditions, parameter values are given in Table 3. 


\section{Conclusion}

In this paper, we have considered a mathematical model of alcohol abuse consisting of four population classes, namely, moderate and occasional drinkers, heavy drinkers, drinkers in treatment and temporarily recovered class. Here, we have found $R_{0}$ as basic reproduction number of the model system (1). In this model the basic reproduction number is the transmission coefficient from moderate and occasional drinker to heavy drinker $\left(\beta_{1}\right)$ divided by the sum of the natural death rate of the population $(\mu)$, the drinking related death rate of heavy drinkers who are not in treatment $\left(\delta_{1}\right)$ and the proportion of individuals who enter treatment $(\phi)$, i.e.,

$$
R_{0}=\frac{\beta_{1}}{\mu+\delta_{1}+\phi} .
$$

Sensitivity analysis of $R_{0}$ identifies $\beta_{1}$, the transmission coefficient from moderate and occasional drinker to heavy drinker, as the most useful parameter to $R_{0}$. Then we have discussed the stability analysis of the model using the basic reproduction number. We have found that the model (1) is locally asymptotically stable at disease free or problem free equilibrium (DFE) $E_{0}$ when $R_{0}<1$. When $R_{0}=1$, a backward bifurcation can occur if $X>\Gamma$ (see 11a,11b, art.7). In that case although $R_{0}$ may be less than 1 , three equilibria can exists together, among which the disease free equilibrium (DFE) and an endemic equilibrium are stable and another endemic equilibrium is unstable. When $R_{0}>1$, analysis produces a quadratic equation of $\mathrm{D}$. The existence of an endemic equilibrium (or endemic equilibria) depends on the existence of at least one real positive value for $\mathrm{D}$. In this case endemic equilibrium exists and becomes stable and the DFE becomes unstable. That means forward bifurcation occurs. In epidemic models, there are two distinct bifurcations at $R_{0}=1$ : (i) forward (supercritical) bifurcation and (ii) backward (subcritical) bifurcation [2, 3, 13, 31, 32, 36, 37]. A forward bifurcation happens when $R_{0}$ crosses unity from below; a small positive asymptotically stable equilibrium appears and the disease-free equilibrium losses its stability. On the other hand, a backward bifurcation happens when $R_{0}$ is less than unity. In this case a small positive unstable equilibrium appears while the disease-free equilibrium and a large positive equilibrium are locally asymptotically stable. Epidemiologically, a backward bifurcation says that, it is not enough to only reduce the basic reproduction number $R_{0}$ to less than one to eliminate a disease and that when $R_{0}$ crosses unity, hysteresis takes place. The stability analysis of endemic equilibrium produces that if the Routh-Hurwitz [17] criterion are satisfied the endemic equilibrium $E^{*}$ is locally asymptotically stable and if $R_{0}<1$ and $\left(\mu+\delta_{1}\right) \geq \beta_{1}$ the disease-free equilibrium (DFE) $E_{0}$ is globally asymptotically stable. Next all our important mathematical findings are numerically verified with the help of MATLAB which supports our analytical results.

The mathematical model presented in this paper shows backward bifurcation which occurs because of the insufficient capacity for treatment policies. As a 
result the drinkers in treatment come to the direct contact of heavy drinkers and they re-enter into the heavy drinkers class. It shows that prevention and control efforts that include treatment and education programmes are in need of improvement so that the rate of relapse from treatment can be reduced. Alcoholism is one of the most common psychiatric disorders with a prevalence. The incidence of alcoholism is still more common in men. But it has been increasing in women. The female to male ratio for alcohol dependence has narrowed to one to two [23]. So an important effect we want to include in our future work is the male/female distribution of alcohol abusers. As part of future work the model considered here can be refined to incorporate drinkers who start regular drinking on their own without having contact with heavy drinkers, age structure and recruitment by drinkers. The mathematical model presented in this paper should be treated with circumspection like other papers due to the assumptions made and the difficulty in the estimation of the model parameters.

\section{REFERENCES}

1. L. Arriola, J. Hyman, Lecture notes, Forward and adjoint sensitivity analysis: with applications in Dynamical Systems, Linear Algebra and Optimization, Mathematical and Theoretical Biology Institute, Summer, 2005.

2. F. Brauer, Backward bifurcation of an epidemic model with saturated treatment function, J. Math. Anal. Appl. 348 (2008) 433-443.

3. F. Brauer, C. Castillo-Chavéz, Mathematical Models in Population Biology and Epidemiology, Springer-Verlay, New York, 2001.

4. S. Busenberg, C. Castillo-Chavéz, Interaction, pair formation and force of infection terms in sexually transmitted diseases. In: C. Castillo-Chavéz (ed.) Mathematical Epidemiology, Lecture Notes in Biomathematics 83 (1989) 280-300.

5. J. Carr, Applications of Center Manifold Theory, Springer-Verlag, New York, 1981.

6. C. Castillo-Chavéz, W. Huang, Competitive exclusion in gonorrhea models and other sexually-transmitted diseases, SIAM, J. Appl. Math. 56 (1996) 494-508.

7. C. Castillo-Chavéz, B.Song, Dynamical models of tuberculosis and their applications, Mathematical Biosciences and Engineering,1 (2004) 361-404.

8. Centers for Disease Control and Prevention (2008), Alcohol and Public Health. http://www.cdc.gov/alcohol/index.htm. Cited 29 Apr 2008.

9. Centers for Disease Control and Prevention (2008),Frequently Asked Questions: What do you mean by heavy drinking? http://www.cdc.gov/alcohol/faqs.htm $\sharp 10$. Cited 11 May 2008.

10. Centers for Disease Control and Prevention (2008),Frequently Asked Questions: What does moderate drinking means? http://www.cdc.gov/alcohol/faqs.htm $\sharp 6$. Cited 11 May 2008.

11. Centers for Disease Control and Prevention (2008), General Information on Alcohol Use and health. http://www.cdc.gov/alcohol/quickstats/generalinfo.htm. Cited 1 May 2008.

12. G. Chowell, P. W. Fenimore, M. A. Castillo-Carsow, C. Castillo-Chavéz, SARS out-breaks in Ontario, HOng Kong and Singapore: the role of diagnosis and isolation as a control mechanism, J. Theor. Biol, 224 (2003) 1-8.

13. College Drinking (2008). http://www.collegedrinkingprevention.gov. Cited 11 May 2008.

14. S. M. Garba, A. B. Gumel, M. R. Abu Bakar, Backward bifurcations in dengue transmission dynamics, Mathematical Biosciences 215 (2008) 11-25.

15. H. Hethcote, The mathematics of infectious disease, SIAM Rev. 42 (2000) 599-653. 
16. H. Hethcote, J. Yorke, Gonorrhea Transmission Dynamics and Control, Lecture Notes in Biomathematics, Springer-Verlag, Berlin 56, 1984.

17. M. Kot, Elements of Mathematical Ecology, Cambridge, Cambridge University Press, 2001.

18. J. Liu, T. Zhang, Global behaviour of a heroin epidemic model with distributive delays, Applied Mathematics Letters 24 (2011) 1685-1692.

19. A. Mubayi, P. Greenwood, C. Castillo-Chavéz, P.J.Gruenewald, D.M.Gorman,On the impact of Relative Residence Times, in highly distinct environments, on the distribution of heavy drinkers, Socio. Econ. Plan. Sci.(In Press).

20. G. Mulone, B. Straughan, A note on heroin epidemics, Mathematical Biosciences 218 (2009) 138-141.

21. National Institute of Alcohol Abuse and Alcoholism (2008) Five Year Strategic Plan. http://www.niaaa.nih.gov/publications/srtategicplan/NIAAASTRATEGICPLAN.htm. Cited 29 Apr 2008.

22. National Institute of Alcohol Abuse and Alcoholism (2008) Frequently Asked Questions for the General Public. http://www.niaaa.nih.gov/FAQs/General-English/default.htm. Cited 29 Apr 2008.

23. NHS Information Centre. http://www.ic.nhs.uk/wefiles/publications. Cited 26 May 2011.

24. F. Nyabadza, S. D. Hove-Musekwa, From heroin epidemics to methamphetamine epidemics: Modelling substance abuse in a South African province, Mathematical Biosciences 225 (2010) 132-140.

25. J. Orford, M. Krishnan, M. Balaam, M. Everitt, K. Van der Graaf, University student drinking: the role of motivational and social factors, Drug-Educ. Prev. Polic. 11 (2004) 407-421.

26. C. Parry, Substance abuse trends in the Western Capes: Summary (25/2/05), Alcohol and Drug Abuse Research Unit, Medical Research Council, 2005.

27. P E. Renshaw, Modelling Biological Populations in Space and Time, Cambridge University Press, Cambridge, 1991

28. G. P. Samanta, Dynamic behaviiour for a nonautonomous heroin epidemic model with time delay, J. Appl. Math. Comput. 35 (2009) 161-178.

29. F. Sánchez, Studies in Epidemiology and Social Dynamics, Dissertation, Cornell University, 2006.

30. F. Sánchez, X. Wang, C. Castillo-Cahvez, D. M.Gorman, P.J. Gruenwald, Drinking as an epidemic: a simple mathematical model with recovery and relapse,In: K. Witkiewitz, G.A. Marlett,(eds.) Therapist's Guide to Evidence- Based Relapse Prevention: Practical Resources for the Mental Health Professional, Academic Press, Burlington (2007) 353-368.

31. O.Sharomi, C.N. Podder, A. B. Gumel, E. H. Elbasha, J. Watmough, Role of incidence function in vaccine-induced backward bifurcation in some HIV models, Mathematical Biosciences 210 (2007) 436-463.

32. B. Song, Seminar Notes, Backward or Forward at $R_{0}=1$, Mathematical and Theoretical Biology Institute, Summer, 2005.

33. P. Van den Driessche, J. Watmough, Reproduction numbers and sub-threshold endemic equilibria for compartmental models of disease transmission, Mathematical Biosciences 180 (2002) 29-48

34. W. Wang, Backward bifurcation of an epidemic model with treatment, mathematical Biosciences 201 (2006) 58-71.

35. E. R. Weitzman, A. Flokman, K.L.Folkman, H. Weschler, The relationship of alcohol outlet density to heavy and frequent drinking and drinking related problems among college students at eight universities, Health Place (2003) 1-6.

36. E. White, C. Comiskey, Heroin epidemics, treatment and ODE modelling, Mathematical Biosciences 208 (2007) 312-324.

37. X. Zhang, X. Liu, Backward bifurcation of an epidemic model with saturated treatment function, J. Math. Anal. Appl. 348 (2008) 433-443. 
Swarnali Sharma got her MSc in Applied Mathematics from Jadavpur University in 2010. Her areas of research are Mathematical Ecology and Epidemiology. She is now working as an Assistant Professor in the Department of Mathematics, Heritage Institute of Technology, Kolkata, 700107, India and pursuing her research work under the supervision of Dr. G.P. Samanta in the Department Of Mathematics, Bengal Engineering and Science University, Shibpur, Howrah, 711103, India.

Present address: Department of Mathematics, Heritage Institute of Technology, Kolkata700107, India.

e-mail: swarnali.sharma87@gmail.com

G. P. Samanta got his MSc and Ph.D. in Applied Mathematics from Calcutta University in 1985 and 1991 respectively. He was a Premchand Raychand Scholar of Calcutta University and received Mouat Medal at the convocation of Calcutta University in 1996. His areas of research are Mathematical Ecology and Operations Research. He is now working as Professor in the Department of Mathematics, Bengal Engineering and Science University, Shibpur, Howrah, 711103, India.

Present address: Department of Mathematics, Bengal Engineering and Science University, Shibpur, Howrah-711103, India.

e-mail: g_p_samanta@yahoo.co.uk 